\title{
Primary Clear Cell Microcystic Adenoma of the Sinonasal Cavity: Pathological or Fortuitous Association?
}

\author{
Rosalin Cooper, ${ }^{1}$ Hannah Markham, ${ }^{1}$ Jeffery Theaker, ${ }^{1}$ Adrian Bateman, ${ }^{1}$ David Bunyan, ${ }^{2}$ \\ Matthew Sommerlad, ${ }^{1}$ Gillian Crawford, ${ }^{3,4}$ and Diana Eccles ${ }^{3,4}$ \\ ${ }^{1}$ Department of Cellular Pathology, University Hospital Southampton NHS Foundation Trust, Southampton, UK \\ ${ }^{2}$ Wessex Regional Genetics Laboratory, Salisbury NHS Foundation Trust, Salisbury, UK \\ ${ }^{3}$ University of Southampton, Southampton, UK \\ ${ }^{4}$ Wessex Clinical Genetics Service, University Hospitals Southampton NHS Foundation Trust, Southampton, UK
}

Correspondence should be addressed to Rosalin Cooper; rosalinanisha.cooper@uhs.nhs.uk

Received 13 August 2016; Revised 14 December 2016; Accepted 15 January 2017; Published 5 February 2017

Academic Editor: Adriana Handra-Luca

Copyright (C) 2017 Rosalin Cooper et al. This is an open access article distributed under the Creative Commons Attribution License, which permits unrestricted use, distribution, and reproduction in any medium, provided the original work is properly cited.

\begin{abstract}
Primary clear cell microcystic adenoma of the sinonasal cavity is rare. It has previously been described only as a VHL-associated tumour. Von Hippel-Lindau (VHL) syndrome is an inherited cancer syndrome characterised by an elevated risk of neoplasia including clear cell renal cell carcinoma (ccRCC), haemangioblastoma, and phaeochromocytoma. We describe the second reported case of a primary clear cell microcystic adenoma of the sinonasal cavity. The 39-year-old patient with VHL syndrome had previously undergone resection and ablation of ccRCC. He presented with epistaxis. Imaging demonstrated a mass in the ethmoid sinus. Initial clinical suspicion was of metastatic ccRCC. However, tumour morphology and immunoprofile were distinct from the previous ccRCC and supported a diagnosis of primary microcystic adenoma. Analysis of DNA extracted from sinonasal tumour tissue did not show loss of the wild-type allele at the VHL locus. Although this did not support tumour association with VHL disease, it was not possible to look for a loss-of-function mutation. The association of primary microcystic adenoma of the sinonasal cavity with VHL disease remains speculative. These lesions are benign but are likely to require regular surveillance. Such tumours may require repeated surgical excision.
\end{abstract}

\section{Introduction}

Von Hippel-Lindau (VHL) disease is a cancer syndrome characterised by an increased risk of multiple tumour types occurring across different organ systems. These include haemangioblastomas within the central nervous system and characteristic visceral lesions including clear cell renal cell carcinoma (ccRCC) and phaeochromocytoma [1]. Microcystic pancreatic lesions can also occur in the context of VHL disease [2]. Affected individuals carry germline mutations in the VHL tumour-suppressor gene with loss of the wild-type allele (normal gene copy) in a VHL disease-associated organ system leading to tumour formation $[1,3]$.

Sinonasal tumours are rare $[4,5]$. Common benign lesions include inverted papillomas and osteomas [4], whilst common malignant lesions include squamous cell carcinoma and adenocarcinoma [5]. There has been only one reported case of a primary clear cell microcystic adenoma of the sinonasal cavity in the literature [6]. In this previously reported case, molecular analysis of tumour DNA confirmed tumour VHL disease association. Here we describe the second reported case of primary clear cell microcystic adenoma of the sinonasal cavity.

\section{Case Report}

2.1. Clinical History. A 39-year-old man with molecularly confirmed VHL disease presented with epistaxis in 2012. Molecular analysis had confirmed a germline VHL mutation in 2004. He had previously undergone excision of cerebellar haemangioblastoma in 2003. He had also undergone bilateral nephron sparing surgery for ccRCC (stage pTla, Fuhrman grade up to 3) in 2005 and renal radiofrequency ablation in both 2008 and 2012. CT imaging demonstrated an ethmoid 


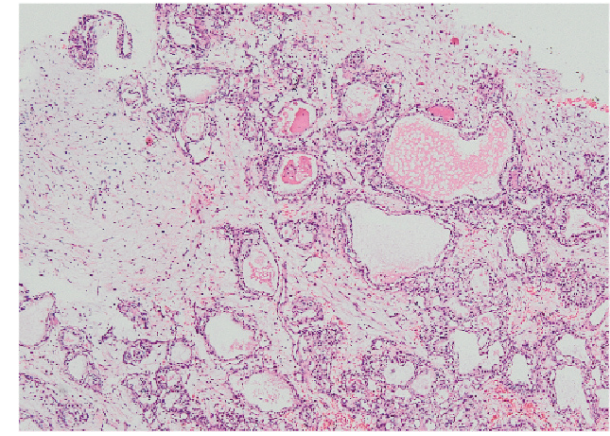

(a)

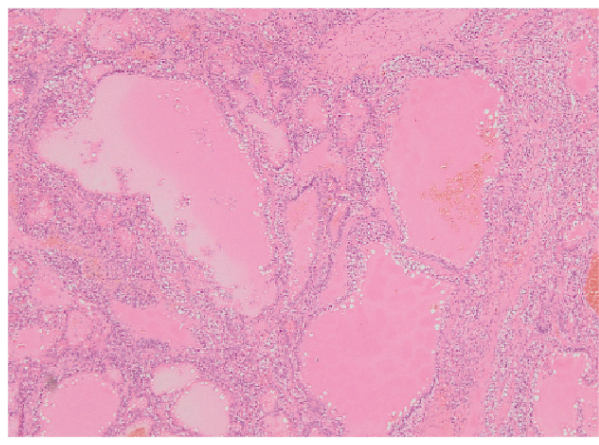

(c)

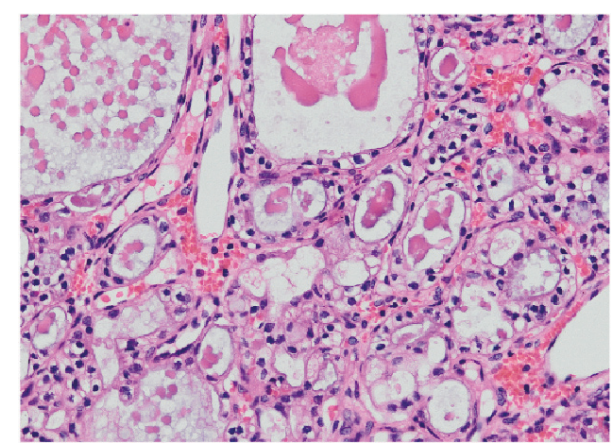

(b)

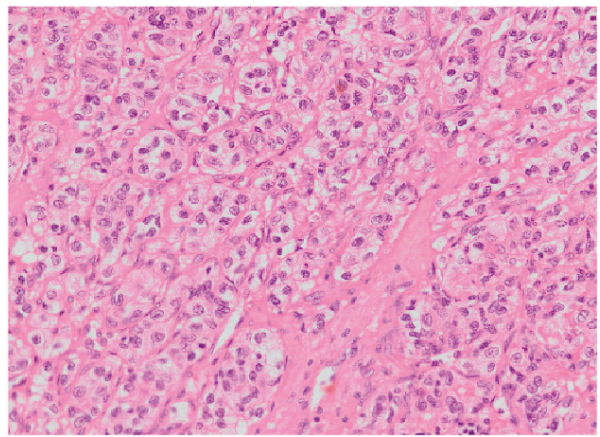

(d)

FIGURE 1: Haematoxylin and eosin (HE) stains of the sinonasal tumour (a) $\times 4$ and (b) $\times 20$ and renal tumour (c) $\times 4$ and (d) $\times 20$.

mass measuring $35 \times 34 \times 44 \mathrm{~mm}$. The patient underwent endoscopic transsphenoidal resection of the lesion. Staging investigations were negative for metastatic disease.

Two years later, in 2014, the patient developed symptoms of nasal obstruction. Sinonasal tumour recurrence was suspected. Imaging demonstrated limited progression in the nasal cavity. Excision biopsy was performed. Histology appeared consistent with the initial presenting sinonasal tumour. Excision appeared complete, with no evidence of residual tumour. Subsequently, symptoms recurred. Therapeutic excision was performed in 2015 and again in 2016. To date, the patient's VHL disease remains stable with no evidence of new lesions or metastatic disease.

The patient remained under the management of specialist multidisciplinary (MDT) team which coordinated regular imaging surveillance and clinical review. The MDT recommended regular magnetic resonance imaging (MRI) surveillance with localised resection as applicable. More extensive surgery or adjuvant therapy was not felt to be indicated.

2.2. Histology. Slides of the sinonasal tumour resected in 2012 and the patient's ccRCC resected in 2005 were collated to allow comparison. Immunohistochemical and special stains for CD10, RCC, CK7, CK20, epithelial marker AE1/3, vimentin, EMA, Ki67, ssms1, sma, alpha-inhibin, p63, thyroglobulin, NSE, s100, GFAP, and PAS were performed according to standard automated protocols (Dako, UK).

The sinonasal tumour demonstrated tubulocystic morphology with a brush border, glycogen-rich cells, and low grade nuclei without conspicuous mitoses (Figures 1(a) and 1(b)). The renal tumour consisted of clear tumour cells with tubulopapillary morphology with hobnailing at the luminal surface with Fuhrman grade up to 3 (Figures 1(c) and 1(d)).

The sinonasal tumour was positive for CK7, epithelial marker AE1/3, vimentin, NSE, and EMA, with patchy CK20 staining, but negative for RCC and CD10. Secretions were PAS-positive and Ki67 demonstrated a low proliferation index. Immunohistochemistry revealed renal tumour positivity for RCC, CD10, and EMA and negativity for CK7 and CK20 (Figures 2 and 3 and Table 1).

2.3. VHL Sequencing Analysis. The patient was known to have a constitutional deletion of exon 1 of $V H L$ confirmed by germline DNA analysis in 2004. DNA extracted from the sinonasal tumour resected in 2012 underwent molecular analysis in 2015 for loss of heterozygosity (LOH) at the VHL locus.

Point mutation analysis of the three coding exons of VHL (Genbank accession number NM_000551.3) was carried out by direct sequencing analysis of sinonasal FFPEextracted tumour DNA. PCR products were generated using a PCR reaction with a $25 \mu \mathrm{l}$ volume and a $60^{\circ} \mathrm{C}$ annealing temperature using exon primers; 1 F-tccgacccgcggatccc, 1R-tcagaccgtgctatcgtcc, 2F-gacgaggtttcaccacgtta, 2R-tcaagtggtctatcctgtact, 3F-tcgttccttgtactgagacc, and 3Rgtaccatcaaaagctgagatg. For the exon 1 fragment $5 \mu$ l of $\mathrm{Q}$ solution was added to the PCR reaction (Qiagen, Manchester, UK). Products were sequenced using the Big-Dye ${ }^{\circledR}$ Terminator v1.1 Cycle Sequencing Kit standard protocol (Applied Biosystems, USA) and separated on an ABI 3130xl Genetic 


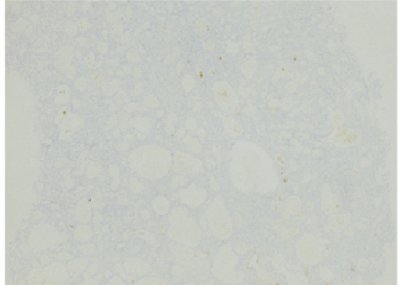

(a)

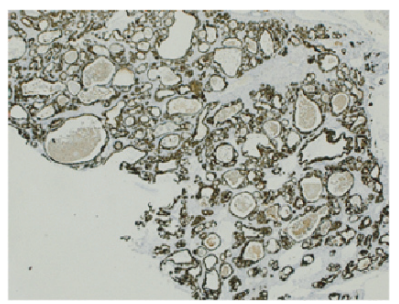

(e)

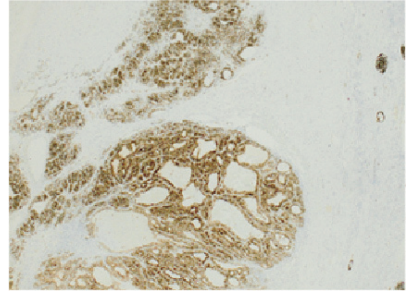

(b)

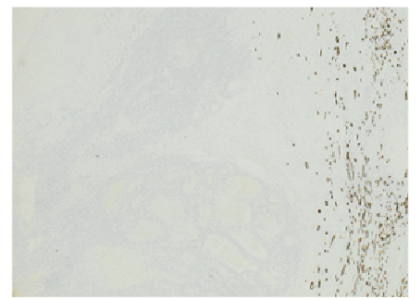

(f)

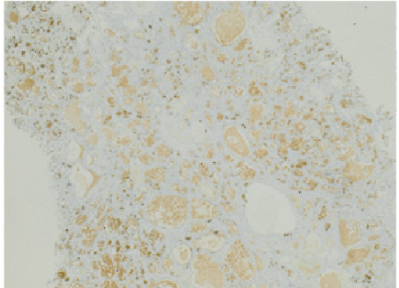

(c)

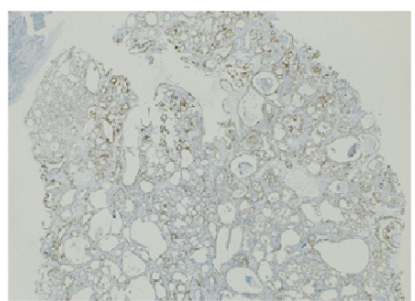

(g)

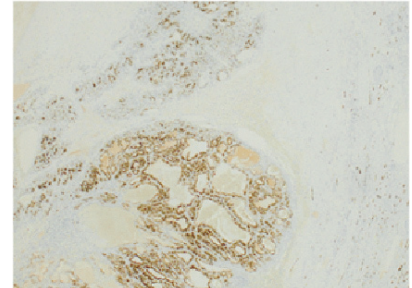

(d)

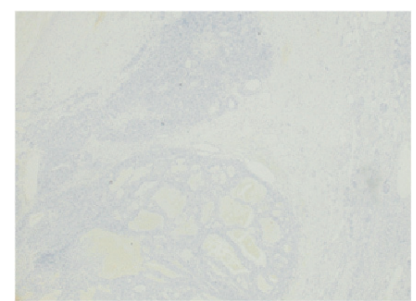

(h)

FIGURE 2: Immunohistochemistry (IHC) of renal and sinonasal tumours, $\times 2$. (a) Sinonasal tumour cells were negative for CD10. (b) Renal tumour cells were strongly positive for CD10. (c) Sinonasal tumour cells were negative for RCC; however, weak staining of luminal secretions was seen. (d) Renal tumour cells demonstrated strong positivity for RCC marker. (e) Sinonasal tumour cells demonstrated positivity for CK7. (f) The renal tumour was negative for CK7; however, in this image a proximal renal tubule was seen to exhibit CK7 positivity. (g) The sinonasal tumour exhibited patchy CK20 positivity. (h) The renal tumour was negative for CK20.

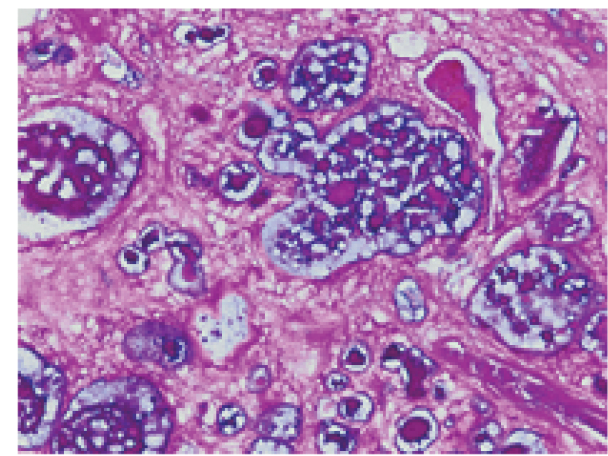

(a)

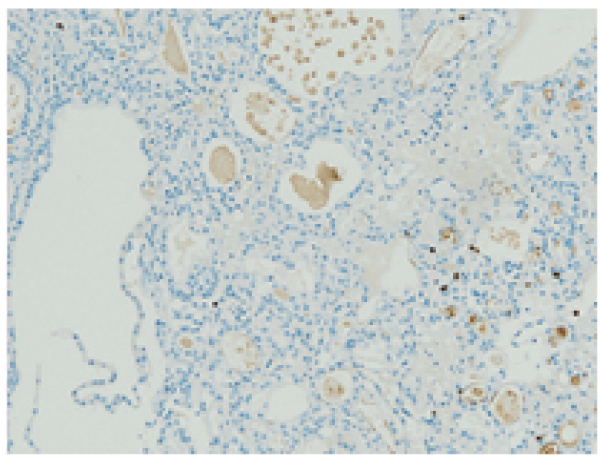

(b)

Figure 3: (a) Sinonasal tumour with luminal PAS-positive secretions, $\times 20$. (b) Ki67 staining demonstrates a low mitotic index, $\times 10$.

Analyzer (Applied Biosystems, USA). Data was analysed using Mutation Surveyor (version 3.1) software (SoftGenetics, USA). Dosage analysis was attempted using the Multiplex Ligation-dependent Probe Amplification (MLPA) method [7], but this failed due to sample quality.

Analysis of tumour DNA demonstrated no causative mutations; however, the tumour was heterozygous for an intron 1 polymorphism (c.341-50G $>$ A), reducing the likelihood of LOH.

\section{Discussion}

Primary tumours of the nasal cavity are rare $[4,5]$. Metastases from other primary tumour sites, whilst unusual, may also occur in this region, including those from a primary ccRCC [8-10]. To date, there has been one reported case of a primary clear cell microcystic adenoma occurring within the sinonasal cavity. This occurred in the context of VHL disease [6].

In the case we present here the initial clinical suspicion was of metastatic ccRCC. Histologically, the clear tumour cells, cystic morphology, and positive staining for AE1/3 [11] and vimentin [12] were supportive of this diagnosis. However, CD10 and RCC negativity, both of which were expressed by the previously resected renal tumour, was not consistent with this interpretation [13]. The tubulocystic morphology, brush border, and presence of PAS positive secretions were also distinct from ccRCC. Furthermore, the tubulocystic morphology, low Ki67 index, and absence of conspicuous mitoses were dissimilar from a primary sinonasal clear cell carcinoma. Sinonasal clear cell carcinomas are rare. These tumours tend to form sheets without glandular structures [14]. They can be locally invasive [15]. Such tumours may also metastasize [15]. 
TABLE 1: Immunohistochemical profile of the renal and sinonasal tumours.

\begin{tabular}{lcc}
\hline $\begin{array}{l}\text { Immunohistochemical } \\
\text { marker }\end{array}$ & $\begin{array}{c}\text { Clear cell } \\
\text { RCC (2005) }\end{array}$ & $\begin{array}{c}\text { Sinonasal tumour } \\
(2012)\end{array}$ \\
\hline CD10 & Positive & Negative \\
RCC & Positive & Negative \\
CK7 & Negative & Positive \\
CK20 & Negative & $\begin{array}{c}\text { Positive (patchy } \\
\text { staining) }\end{array}$ \\
EMA & Positive & Positive \\
Ki67 & & Low proliferation \\
Epithelial marker AE1/3 & & Positive \\
Vimentin & & Positive \\
ssms1 & & Negative \\
sma & & Negative \\
Alpha-inhibin & & Negative \\
p63 & & Negative \\
Thyroglobulin & & Negative \\
NSE & & Positive \\
S100 & Negative \\
GFAP & Negative \\
PAS & & positive \\
\hline
\end{tabular}

In the previously reported case of a primary clear cell microcystic adenoma of the nasal sinus, Xu et al. describe microcystic tumour morphology, clear glycogen-rich cells without nuclear atypia, EMA and cytokeratin positivity, and negativity for CD10 [6]. These features are consistent with those of the case presented here. Similarity was also noted between features of the sinonasal tumour and those of pancreatic microcystic adenoma. Typically these pancreatic tumours, which can be both VHL-associated and sporadic, are characterised by cystic morphology, glycogenrich cytoplasm, a low mitotic index, and AE1/3 positivity [2]. These are benign lesions [2]. Conventionally, small lesions in asymptomatic patients managed conservatively with regular imaging surveillance. Surgical resection is considered for larger or symptomatic tumours [16]. It is possible that the primary microcystic adenoma of the sinonasal tract is a similar tumour entity.

$\mathrm{Xu}$ et al. demonstrated sinonasal microcystic adenoma VHL LOH [6]. This confirmed tumour association with VHL disease [6]. In the case we describe here the sinonasal tumour was heterozygous at the VHL locus. The presence of an intron 1 polymorphism makes $\mathrm{LOH}$ unlikely. Therefore, molecular analysis did not support tumour association with VHL disease. However, it was not possible to test for a loss-of-function tumour mutation. It is also feasible that heterogeneity of the tumour means that this result is not truly representative of $\mathrm{LOH}$ status.

In the World Health Organisation (WHO) classification of head and neck tumours, adenomatous lesions of the sinonasal cavity are of salivary gland origin [17]. However, benign microcystic lesions similar to the case reported here are not described within the current classification. Due to the rarity of this tumour entity and uncertainty regarding its biological history, categorisation under the current WHO classification is not meaningful. In view of the morphological features, immunoprofile, and similarities to microcystic lesions in the pancreas and previously reported case we feel that the lesion is best described as a microcystic adenoma.

The sinonasal microcystic adenoma described by $\mathrm{Xu}$ et al. was surgically resected due to local progression. However, the clinical course of such tumours is unknown [6]. In the case described here, following initial surgical excision, to date three further therapeutic excisions have been required due to local recurrence. The rapid local recurrence is suggestive of a fast-growing lesion, despite the low mitotic index. Therefore, regular imaging surveillance may be indicated, and surgical resection may be appropriate.

\section{Conclusions}

In conclusion, the association of primary microcystic adenoma of the sinonasal cavity with VHL disease is possible but not proven. Such lesions are rare and may provide a considerable diagnostic challenge. Importantly, these lesions are considered essentially benign. However, they may have potential for rapid growth and local recurrence. This suggests that in such cases regular surveillance is merited and that repeated surgical resection may be required.

\section{Consent}

Written informed patient consent was obtained regarding the publication of this case.

\section{Disclosure}

This case was presented as a poster presentation at Nottingham Pathology 2016, the 9th Joint Meeting of the British Division of the International Academy of Pathology and the Pathological Society of Great Britain \& Ireland in June 2016. Therefore, a modified abstract of this case was published in the Journal of Pathology On-Line Supplement in October 2016 (J Pathol 2016; 240 Suppl 1: S35).

\section{Competing Interests}

The authors declare that there is no conflict of interests regarding the publication of this paper

\section{Acknowledgments}

The authors acknowledge the valuable contribution of Professor Stewart Fleming in the assessment of this case.

\section{References}

[1] R. R. Lonser, G. M. Glenn, M. Walther et al., "Von HippelLindau disease," Lancet, vol. 361, no. 9374, pp. 2059-2067, 2003. 
[2] V. H. Mohr, A. O. Vortmeyer, Z. Zhuang et al., "Histopathology and molecular genetics of multiple cysts and microcystic (serous) adenomas of the pancreas in von Hippel-Lindau patients," The American Journal of Pathology, vol. 157, no. 5, pp. 1615-1621, 2000.

[3] A. G. Knudson Jr. and L. C. Strong, "Mutation and cancer: neuroblastoma and pheochromocytoma," American Journal of Human Genetics, vol. 24, no. 5, pp. 514-532, 1972.

[4] B. Schick and J. Dlugaiczyk, "Benign tumors of the nasal cavity and paranasal sinuses," in Rhinology and Facial Plastic Surgery, F. J. Stucker, C. De Souza, G. S. Kenyon et al., Eds., pp. 377-385, Springer, Berlin, Germany, 2009.

[5] G. G. Calzada and R. S. Weber, "Malignant tumors of the nose and paranasal cavity," in Rhinology and Facial Plastic Surgery, F. J. Stucker, C. De Souza, G. Kenyon et al., Eds., pp. 387-394, Springer, Berlin, Germany, 2009.

[6] D. S. Xu, M. S. Dirks, M. M. Quezado et al., "A von HippelLindau disease-associated microcystic adenoma of the ethmoid sinus: case report," Neurosurgery, vol. 69, no. 4, pp. E1017-E1022, 2011.

[7] J. P. Schouten, C. J. McElgunn, R. Waaijer, D. Zwijnenburg, F. Diepvens, and G. Pals, "Relative quantification of 40 nucleic acid sequences by multiplex ligation-dependent probe amplification," Nucleic acids research, vol. 30, no. 12, p. e57, 2002.

[8] H. M. Lee, H. J. Kang, and S. H. Lee, "Metastatic renal cell carcinoma presenting as epistaxis," European Archives of OtoRhino-Laryngology, vol. 262, no. 1, pp. 69-71, 2005.

[9] A. Remenschneider, P. Sadow, D. Lin, and S. Gray, "Metastatic renal cell carcinoma to the sinonasal cavity: a case series," Journal of Neurological Surgery Reports, vol. 74, no. 2, pp. 6772, 2013.

[10] T. Terada, "Renal cell carcinoma metastatic to the nasal cavity," International Journal of Clinical and Experimental Pathology, vol. 5, no. 6, pp. 588-591, 2012.

[11] N. Weinbreck, B. Marie, A. Bressenot et al., "Immunohistochemical markers to distinguish between hemangioblastoma and metastatic clear-cell renal cell carcinoma in the brain: utility of aquaporinl combined with cytokeratin AE1/AE3 immunostaining," American Journal of Surgical Pathology, vol. 32, no. 7, pp. 1051-1059, 2008.

[12] B. F. Skinnider, A. L. Folpe, R. A. Hennigar et al., "Distribution of cytokeratins and vimentin in adult renal neoplasms and normal renal tissue: potential utility of a cytokeratin antibody panel in the differential diagnosis of renal tumors," The American Journal of Surgical Pathology, vol. 29, no. 6, pp. 747-754, 2005.

[13] A. K. Avery, J. Beckstead, A. A. Renshaw, and C. L. Corless, "Use of antibodies to RCC and CD10 in the differential diagnosis of renal neoplasms," The American Journal of Surgical Pathology, vol. 24, no. 2, pp. 203-210, 2000.

[14] T. Imai, I. Satoh, K. Matsumoto et al., "Clear cell carcinoma of the nasal cavity: a case report from histopathological viewpoint," Auris Nasus Larynx, vol. 43, no. 1, pp. 108-111, 2016.

[15] W. Zhao, L. Yang, L. Wang et al., "Primary clear cell carcinoma of nasal cavity: report of six cases and review of literature," International Journal of Clinical and Experimental Medicine, vol. 7, no. 12, pp. 5469-5476, 2014.

[16] J. F. Tseng, A. L. Warshaw, D. V. Sahani et al., "Serous cystadenoma of the pancreas: tumor growth rates and recommendations for treatment," Annals of Surgery, vol. 242, no. 3, pp. 413421, 2005.
[17] L. Barnes, J. W. Eveson, P. Reichart et al., Eds., World Health Organization Classification of Tumours. Pathology and Genetics of Head and Neck Tumours, IARC Press, Lyon, France, 2005. 


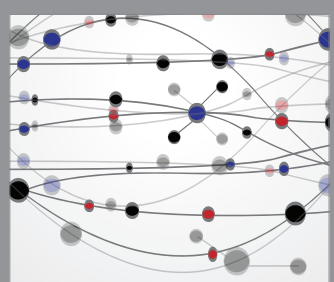

The Scientific World Journal
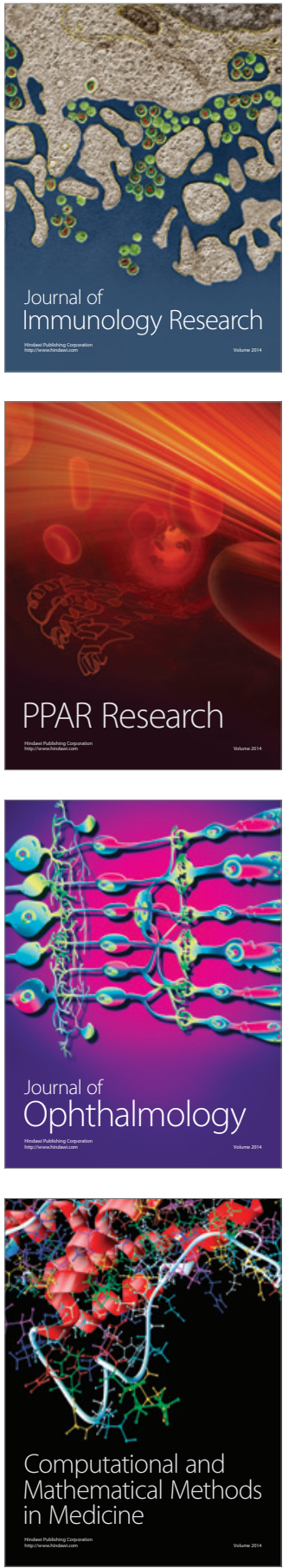

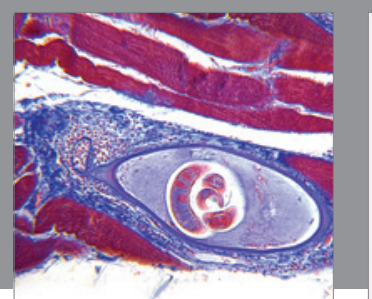

Gastroenterology Research and Practice
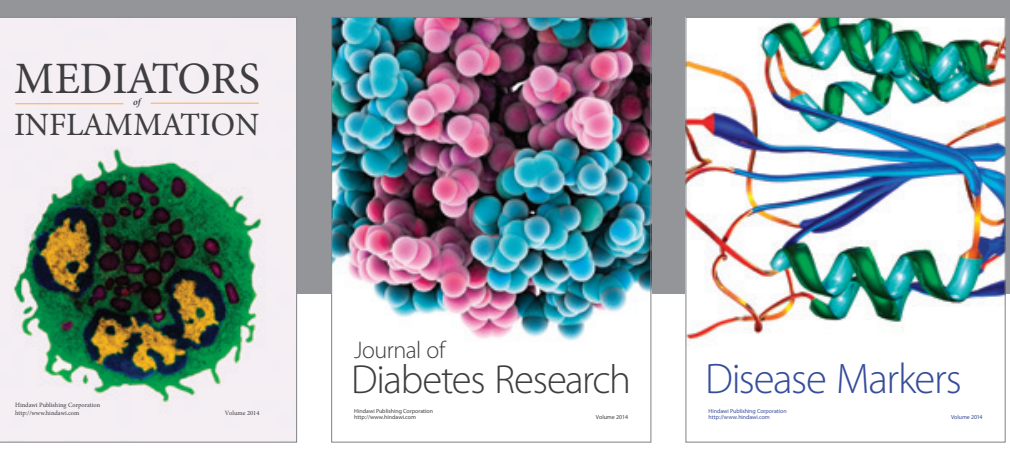

Disease Markers

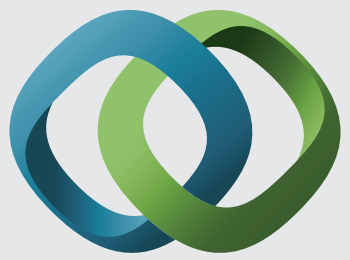

\section{Hindawi}

Submit your manuscripts at

https://www.hindawi.com
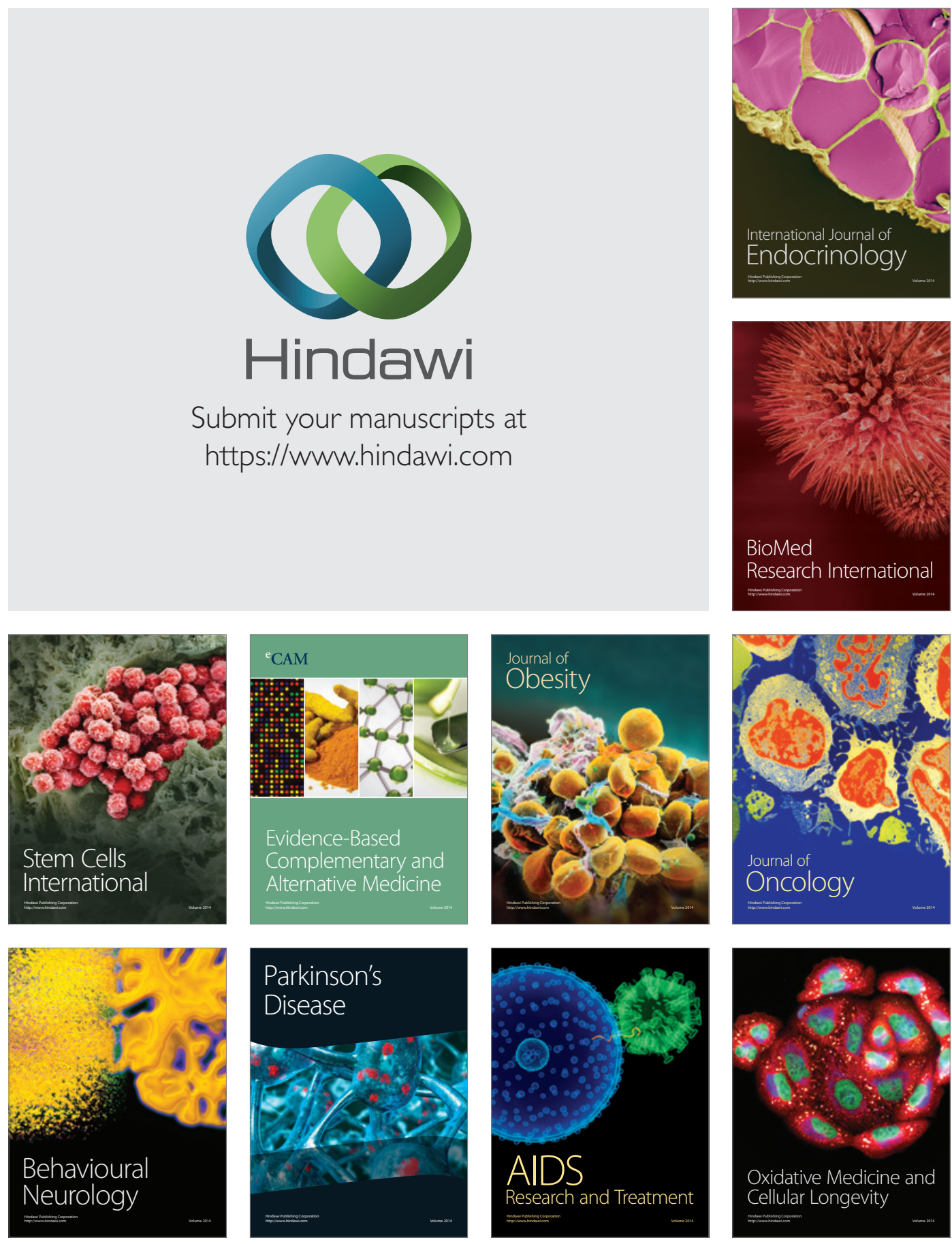